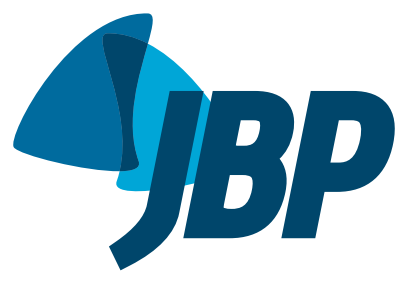

\title{
Gel pillow designed specifically for obstructive sleep apnea treatment with continuous positive airway pressure
}

Adriana Salvaggio', Anna Lo Bue¹, Serena lacono Isidoro', Salvatore Romano ${ }^{1}$, Oreste Marrone' ${ }^{1}$, Giuseppe Insalaco ${ }^{1}$

1. Consiglio Nationale delle Ricerche, Istituto di Biomedicina ed Immunologia Moleculare "Alberto Monroy", Palermo, Italia.

Submitted: 15 January 2016. Accepted: 31 May 2016.

Study carried out at the Consiglio Nazionale delle Ricerche, Istituto di Biomedicina ed Immunologia Molecolare "Alberto Monroy", Palermo, Italia.

\begin{abstract}
Objective: To determine whether the use of a gel pillow with side cutouts designed to accommodate a continuous positive airway pressure (CPAP) mask and reduce head temperature improves the efficacy of and adherence to auto-CPAP therapy. Methods: Twenty-three consecutive CPAP-naive patients with obstructive sleep apnea were enrolled in the study. Patients were given an auto-CPAP machine with an appropriate CPAP mask and were instructed to use CPAP for 15 nights. They were instructed to sleep with their own pillow (the control pillow) from nights 1 to 5 and with either a foam pillow or a gel pillow, both of which had side cutouts, for 5 consecutive nights each, in random order. After night 15, auto-CPAP machine data were downloaded and patients rated their satisfaction with each pillow on a visual analog scale. Results: Twenty-two patients completed the protocol. The pressures administered, residual apnea-hypopnea index, air leaks, and mean duration of CPAP use did not differ among the periods during which each pillow was used. Patients were significantly more satisfied with the gel pillow than with the control pillow and the foam pillow $(p=0.022$ and $p=0.004$, respectively), their level of satisfaction with the gel pillow correlating significantly with excessive daytime sleepiness $\left(r^{2}=0.19 ; p=0.0443\right)$. Conclusions: Among obstructive sleep apnea patients treated with nasal CPAP, the use of a gel pillow with side cutouts appears to have no impact on treatment effectiveness. Nevertheless, such patients seem to prefer a gel pillow over other types of pillows.
\end{abstract}

Keywords: Sleep; Continuous positive airway pressure; Sleep apnea, obstructive; Masks.

\section{INTRODUCTION}

Sleep represents one third of the human life. In addition to improving cognitive performance, mood state, and quality of life, good sleep quality and enough sleep have a major impact on health conditions and life expectancy. ${ }^{(1)}$ It has been demonstrated that short sleep duration is associated with hypertension, stroke, diabetes, and possibly other diseases. ${ }^{(2)}$ Sleep can be disturbed by environmental conditions, such as noise, temperature, and humidity, and can be influenced by several psychiatric, neurological, or clinical disorders. ${ }^{(3)}$ One of the most common sleep disorders is obstructive sleep apnea (OSA), ${ }^{(4)}$ which is characterized by recurrent episodes of complete or partial obstruction of the upper airway during sleep. OSA can cause excessive daytime sleepiness, as well as predisposing to a variety of diseases and reducing life expectancy. ${ }^{(5,6)}$

The first-line treatment of OSA is continuous positive airway pressure (CPAP) therapy administered during sleep, usually through a nasal or oronasal mask, in order to maintain the upper airway fully open. ${ }^{(7)}$ The benefits of CPAP therapy include resolution of respiratory disturbances and an increased amount of sleep during CPAP application. ${ }^{(8)}$
In many OSA patients, adherence to CPAP therapy is less than optimal. (9) Several mechanisms might be responsible for air leak during CPAP therapy. For example, mouth opening can lead to large air leaks, which in turn can cause drying of the oronasal passageway, eye irritation, ${ }^{(10)}$ increased noise, device malfunctioning (particularly if it is an auto-CPAP machine), and, consequently, intolerance to positive airway pressure therapy. Patients commonly complain that the CPAP device is cumbersome and does not allow them to move freely while in bed. In fact, body posture changes can cause mask displacement and excessive air leaks if the mask hits the pillow upon which the head of the patient lies. ${ }^{(11)}$ Therefore, turning in bed can be difficult. In addition, head movements should be avoided, and this can increase the sensation of heat around the head, which can further increase patient discomfort and disrupt sleep. Discomfort during sleep can lead patients to remove the CPAP mask early in the night, and this can lead to the reappearance of respiratory disturbances. The use of comfortable, appropriately shaped pillows during CPAP application could minimize air leaks, improve patient mobility, improve the quality of sleep, and increase CPAP use, thus increasing the benefits of CPAP therapy.

Correspondence to:

Adriana Salvaggio. Consiglio Nazionale delle Ricerche, Istituto di Biomedicina ed Immunologia Molecolare "Alberto Monroy", Via Ugo La Malfa, 153, 90146, Palermo, Italia. Tel.: 39091 6809-143. E-mail: salvaggio@ibim.cnr.it 
We hypothesized that the use of a gel pillow that could accommodate the CPAP mask when patients lie on their side and that could reduce head temperature and sweating ${ }^{(12)}$ would improve the efficacy of and adherence to auto-CPAP therapy. Our primary objective was to determine whether the specially designed pillow was associated with fewer air leaks during auto-CPAP application and fewer respiratory disturbances, as automatically detected by the device. A secondary objective was to determine whether gel pillows with side cutouts were preferred by OSA patients receiving auto-CPAP and whether their use was associated with increased short-term adherence to therapy.

\section{METHODS}

Individuals 18 years of age or older referred to our sleep laboratory for suspected OSA were screened for inclusion in the present study. Out-of-center nocturnal cardiorespiratory polygraphic recordings were performed. Patients with at least moderate OSA (an apnea-hypopnea index $[\mathrm{AHI}] \geq 15$ events/h) were advised to initiate treatment with CPAP and were invited to participate in the study. The first 23 patients who agreed to participate were included in the study. One patient did not complete the study. All 23 lived near the sleep laboratory (i.e., less than 30 $\mathrm{km}$ from it). Sample size was powered to estimate patient satisfaction, expressed as a change of at least 2 points in the visual analog scale (VAS) score, with a power of $90 \%$, an error probability of 0.05 , and an SD of 2 for the change in VAS score. The study protocol was approved by the Research Ethics Committee of the University of Palermo, located in the city of Palermo, Italy. All participating patients gave written informed consent.

After medical history taking, all patients who had psychiatric disorders or diseases that might interfere with the outcomes of CPAP therapy were excluded, as were those who did not give written informed consent. Patients presenting with impairments or comorbidities considered likely to interfere with adherence to instructions, including neuromuscular disease, unstable psychiatric disease, cognitive impairment, myocardial infarction, unstable angina, heart failure, stroke, and lung disease, were excluded.-

\section{Pillows tested}

Three pillows were tested in the present study: each patient's own pillow (control pillow); a foam pillow (Technogel Italia S.r.l., Vicenza, Italy); and a gel pillow produced with a combination of memory foam and a stable, plasticizer-free polyurethane gel (Technogel Italia S.r.l.). The last two pillows had a rectangular design and side cutouts designed specifically for CPAP therapy, and both measured $66 \mathrm{~cm}$ in length $\times 40 \mathrm{~cm}$ in width $\times 14 \mathrm{~cm}$ in height (Figure 1 ).

\section{Protocol}

In all patients, the body mass index was calculated as weight in kilograms divided by height in meters squared $\left(\mathrm{kg} / \mathrm{m}^{2}\right)$. The subjective level of sleepiness was assessed with the Epworth Sleepiness Scale.

Nocturnal cardiorespiratory polygraphic recordings were performed with a Somté recorder (Compumedics Inc., Abbotsford, Australia). Nasal airflow was detected through nasal cannulas and a pressure transducer. Respiratory disturbances were manually analyzed. Apneas were identified by cessation of nasal airflow for at least $10 \mathrm{~s}$ and were defined as obstructive when thoracoabdominal movements persisted. Hypopneas were identified by a $\geq 30 \%$ decrease in the amplitude of the airflow signal for at least $10 \mathrm{~s}$ and $\mathrm{a}>3 \%$ decrease in $\mathrm{SaO}_{2}$.

Respiratory event index was calculated as the number of apneas and hypopneas per hour of monitoring time. The percentage of sleep time spent at $\mathrm{SaO}_{2}<$ $90 \%$ was automatically calculated by the software accompanying the Somté recorder (Compumedics Inc.). In accordance with the recommendations of the American Academy of Sleep Medicine, moderate OSA was defined as an AHI $\geq 15$ events/h, whereas severe OSA was defined as an AHI > 30 events/h, with a predominance of obstructive events. ${ }^{(10)}$

After adaptation to breathing while receiving CPAP therapy, the patients were instructed on how to run an auto-CPAP machine (S9; ResMed, Abingdon, UK) and were requested to use it at home for 15 nights.

All patients were informed of the diagnosis by the medical staff, who, in addition to describing the disease and the consequences of ineffective treatment,
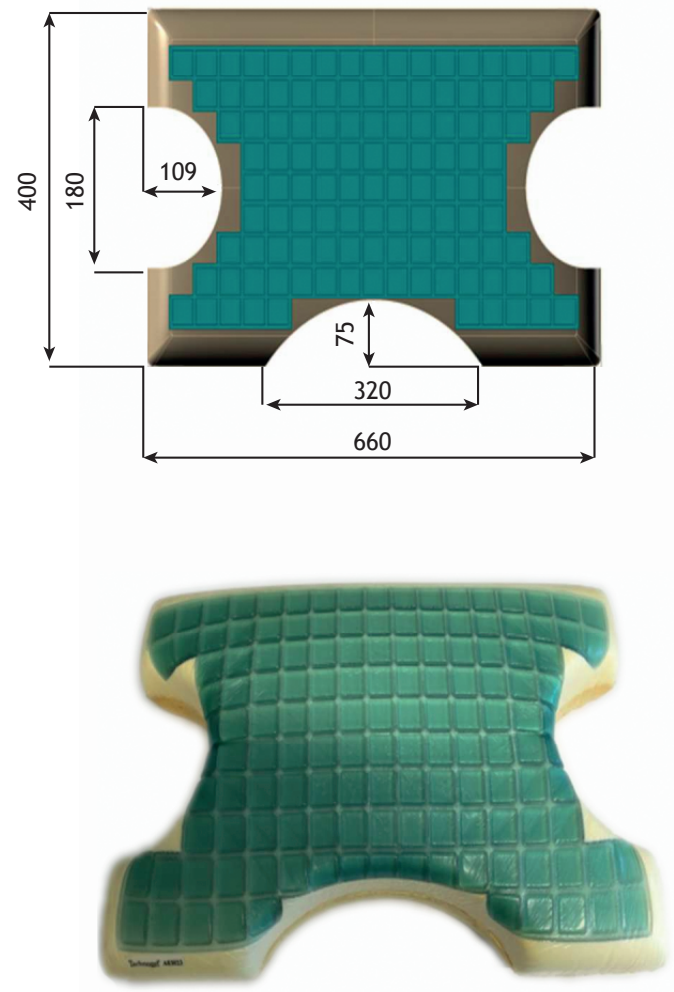

Figure 1. Characteristics of the gel pillow used in the present study. All measurements are expressed in $\mathrm{mm}$. 
provided information regarding the follow-up of patients with OSA. Subsequently, the nursing staff assisted patients in identifying the most suitable mask and held a morning session of education and training on CPAP therapy. In addition, patients were given an oximeter for continuous nocturnal $\mathrm{SaO}_{2}$ recordings and a data logger for monitoring environmental pressure, temperature, and humidity. Patients were instructed to sleep with the control pillow from nights 1 to 5 and with either the foam pillow or the gel pillow from nights 6 to 15 . The order of use of the last two pillows was randomized, with patients starting alternatively with the gel pillow or the foam pillow.

Patients returned all instruments after night 15 . Auto-CPAP machine data were downloaded in order to assess the pressures administered, residual AHI, air leaks, and adherence to CPAP therapy. The recorded $\mathrm{SaO}_{2}$ values and environmental conditions were analyzed. Patients were asked to rate their experience with each of the three pillows by marking a point on a VAS consisting of a $10-\mathrm{cm}$ line, the left and right ends of which corresponded to total dissatisfaction and total satisfaction, respectively.

\section{Statistical analysis}

Means were compared by the nonparametric Kruskal-Wallis test. Linear regression was used in order to assess the relationships between variables. Data are reported as mean \pm SD. Values of $p<0.05$ were considered significant. Statistical analysis was performed with JMP software, version 8.0 (SAS Institute Inc., Cary, NC, USA).

\section{RESULTS}

All patients but one completed the protocol. The characteristics of the 22 patients who completed the protocol are shown in Table 1.

There were no significant differences in environmental temperature, humidity, or barometric pressure among the periods during which each pillow was used. With regard to the outcomes of auto-CPAP therapy, the three periods were similar in terms of residual AHI, delivered pressure, and air leaks (Table 2). However, when the only patient who used an oronasal mask slept with the control pillow, the amount of air leak was highest (21.6 L/min), decreasing significantly with the use of the gel pillow $(6.0 \mathrm{l} / \mathrm{min})$ and the foam pillow (7.2 L/min).

Duration of auto-CPAP use did not differ among pillow types (Table 2) and did not correlate with VAS scores. Figure 2 shows the mean VAS scores for each pillow type. The gel pillow was associated with a significantly higher satisfaction level than were the control pillow ( $p$ $=0.022)$ and the foam pillow $(p=0.004)$. Although the VAS score for the gel pillow was significantly correlated with excessive daytime sleepiness $\left(r^{2}=\right.$ $0.19 ; p=0.0443)$, those for the remaining pillow types were not (Figure 3 ). All participants reported a satisfaction level $>5$ with the gel pillow.

\section{DISCUSSION}

This is the first study to examine whether a particular pillow shape and material are associated with increased efficacy of and adherence to CPAP therapy. CPAP therapy plays an important role in improving health status, prolonging survival, ${ }^{(13)}$ and improving quality of life in patients with OSA. ${ }^{(14)}$ Recent studies have suggested that it is important to maintain CPAP until the last hours of the night, when rapid eye movement sleep is most abundant, given that rapid eye movement-related respiratory disturbances are dangerous because of their health consequences. ${ }^{(15,16)}$ Therefore, measures aimed at improving treatment adherence and efficacy are required.

We found no differences between the gel pillow and the control pillow regarding treatment effectiveness and short-term adherence to treatment. However, the gel pillow was better accepted by the participating patients.

Although the side cutouts in the pillow were designed to eliminate one of the factors responsible for air leaks, they neither reduced air leaks nor improved respiratory disturbances, as well as having had no impact on patient comfort. However, in most of our patients, there was minimal air leak during the night, as well as a low residual AHI. This was possibly due to careful instructions and adaptation to CPAP before initiation of treatment. In addition, all but one of the participating patients used a nasal CPAP mask, which

Table 1. Characteristics of the study sample and results of nocturnal polygraphic recordings. ${ }^{\text {a }}$

\begin{tabular}{cccccc} 
Male/female, $\mathbf{n} / \mathbf{n}$ & Age, years & BMI, $\mathbf{k g} / \mathbf{m}^{2}$ & AHI, $\mathbf{n} / \mathbf{h}$ & TSat $_{90^{\prime}} \%$ & ESS score \\
\hline $19 / 3^{b}$ & $53.3 \pm 7.6$ & $33.7 \pm 6.5$ & $47.1 \pm 19.4$ & $24.0 \pm 23.6$ & $10.3 \pm 4.7$ \\
\hline
\end{tabular}

BMI: body mass index; AHI: apnea-hypopnea index; TSat $_{90}$ : percentage of sleep time spent at $\mathrm{SaO}_{2}<90 \%$; and ESS: Epworth Sleepiness Scale. aData are presented as mean \pm SD, except where otherwise indicated. ${ }^{b} D a t a$ presented as $\mathrm{n}$.

Table 2. Data downloaded from auto-CPAP machines. ${ }^{a}$

\begin{tabular}{lccc}
\multicolumn{1}{c}{ Variable } & Control pillow & Foam pillow & Gel pillow \\
Residual AHI, $\mathrm{n} / \mathrm{h}$ & $3.7 \pm 2.9$ & $2.7 \pm 1.9$ & $2.7 \pm 1.8$ \\
90th percentile pressure, $\mathrm{cmH}_{2} \mathrm{O}$ & $12.3 \pm 2.7$ & $11.8 \pm 2.8$ & $12.2 \pm 2.7$ \\
Air leak, $\mathrm{L} / \mathrm{m}$ & $10.4 \pm 6.2$ & $11.1 \pm 8.5$ & $10.8 \pm 7.0$ \\
Mean duration of use, $\mathrm{min} /$ day & $395 \pm 93$ & $373 \pm 74$ & $386 \pm 80$ \\
\hline
\end{tabular}

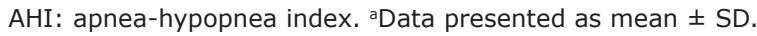


allowed them to turn on their sides easily with no increase in air leak.

Patients with OSA syndrome can generally switch between nasal and oronasal masks without changing machine pressure, although there are individual differences that can be clinically significant. Most patients with OSA syndrome prefer a nasal mask as the interface for initiation of CPAP. However, in comparison with CPAP titration with a nasal mask, CPAP titration with an oronasal mask is characterized by increased mask leak, increased residual respiratory disturbance, increased arousal indices, decreased slow wave sleep, and decreased total sleep time on the titration night. ${ }^{17,18)}$ In the present study, the only patient who used CPAP via an oronasal mask had increased air leak when using the control pillow, air leak being markedly decreased with the use of the foam pillow and the gel pillow. Therefore, it is possible that specially designed pillows are indicated for patients treated with CPAP via an oronasal mask. Studies involving an adequate sample of patients receiving CPAP therapy via an oronasal mask are needed in order to test this hypothesis.

Although the gel pillow was not associated with changes in the efficacy of CPAP therapy in the present study, it improved sleep comfort, particularly in the sleepiest patients. However, the duration of CPAP use tended to be the same irrespective of the type of pillow. Nevertheless, each pillow was tested for only 5 nights. In the long term, improved comfort might result in longer sleep duration and prolonged CPAP use. In fact, many OSA patients have reported that they remove their CPAP mask if they wake up in the middle of the night. A higher degree of comfort during the night might improve sleep continuity and treatment adherence in the long term. It is not clear why the sleepiest patients in the present study were the ones who liked the gel pillow the most. Among

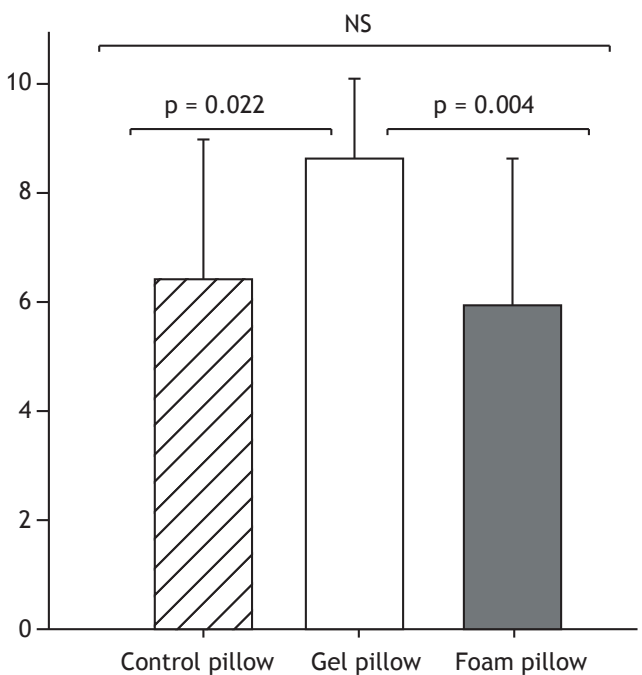

Figure 2. Patient satisfaction with the pillows tested (vertical bars), as expressed by visual analog scale scores. Data are presented as mean \pm SD. NS: not significant.
OSA patients, the sleepiest are those who have the worst quality of life and who are at the highest risk for accidents and health consequences, such as systemic hypertension. (19) On the basis of our findings, we believe that the sleepiest of OSA patients, in whom OSA is typically most severe, should be advised to
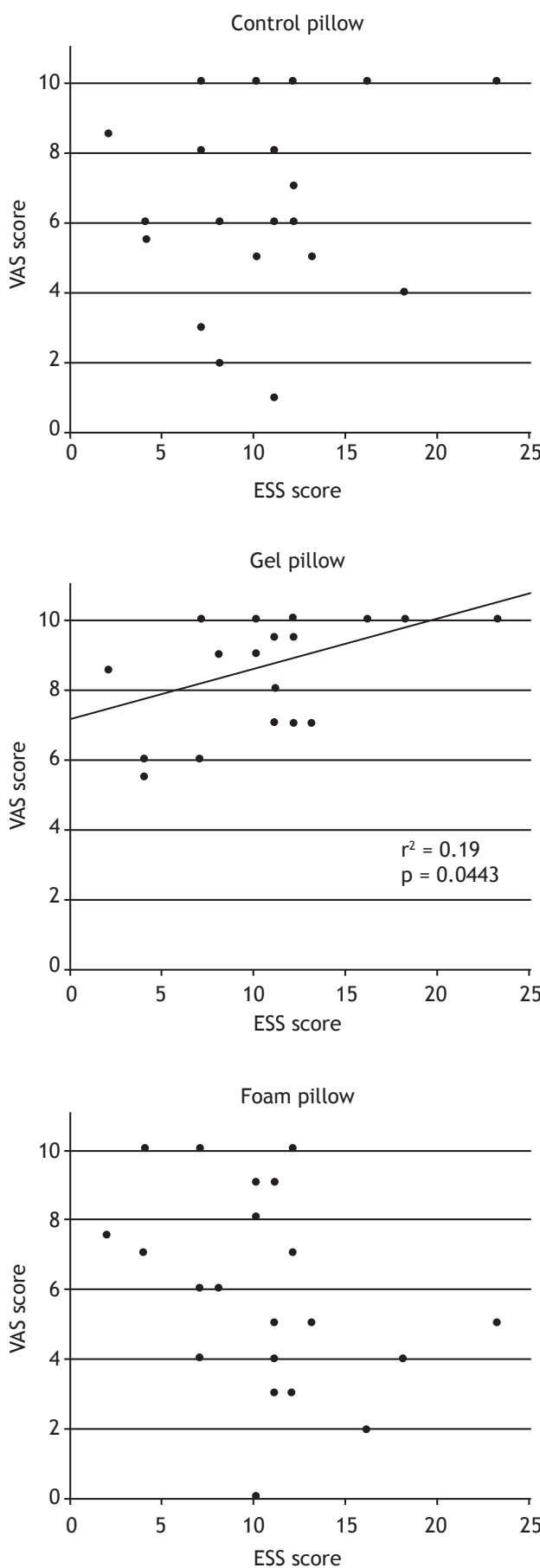

Figure 3. Linear regression between sleepiness, as expressed by Epworth Sleepiness Scale (ESS) scores, and patient satisfaction with the pillows tested, as expressed by visual analog scale (VAS) scores. 
use gel pillows when receiving CPAP, in an attempt to improve treatment adherence.

One limitation of the present study is that only one participant used an oronasal mask for CPAP therapy. Although data for that patient suggest that pillows with side cutouts play a beneficial role in CPAP therapy, further studies are warranted. Another limitation is that we relied on data downloaded from auto-CPAP machines in order to evaluate treatment effectiveness. Such data cannot be exactly reproduced by means of direct assessment; however, according to the American Thoracic Society, they might reflect the results of direct assessment, particularly when they indicate very good or very poor treatment outcomes, ${ }^{(20)}$ as was the case in our patients.

\section{REFERENCES}

1. Crawford-Achour E, Dauphinot V, Martin MS, Tardy M, Gonthier R, Barthelemy JC, et al. Protective Effect of Long-Term CPAP Therapy on Cognitive Performance in Elderly Patients with Severe OSA: The PROOF Study. J Clin Sleep Med. 2015;11(5):519-24. http://dx.doi. org/10.5664/jcsm.4694

2. Torres G, Sánchez-de-la-Torre M, Barbé F. Relationship Between OSA and Hypertension. Chest. 2015;148(3):824-32. http://dx.doi org/10.1378/chest.15-0136

3. Van Deun D, Verhaert V, Willemen T, Wuyts J, Verbraecken J, Exadaktylos $\mathrm{V}$, et al. Biomechanics-based active control of bedding support properties and its influence on sleep. Work. 2012; 41 Suppl 1:1274-80

4. Peppard PE, Young T, Barnet JH, Palta M, Hagen EW, Hla KM Increased prevalence of sleep-disordered breathing in adults. Am J Epidemiol. 2013;177(9):1006-14. http://dx.doi.org/10.1093/aje/ kws342

5. Flemons WW. Clinical practice. Obstructive sleep apnea. N Engl J Med. 2002;347(7):498-504. http://dx.doi.org/10.1056/ NEJMcp012849

6. Stansbury RC, Strollo PJ. Clinical manifestations of sleep apnea. J Thorac Dis. 2015;7(9):E298-310.

7. Epstein LJ, Kristo D, Strollo PJ Jr, Friedman N, Malhotra A, Patil SP et al. Clinical guideline for the evaluation, management and longterm care of obstructive sleep apnea in adults. J Clin Sleep Med. 2009;5(3):263-76.

8. Weaver TE, Maislin G, Dinges DF, Bloxham T, George CF Greenberg $\mathrm{H}$, et al. Relationship between hours of CPAP use and achieving normal levels of sleepiness and daily functioning. Sleep. 2007;30(6):711-9.

9. Wohlgemuth WK, Chirinos DA, Domingo S, Wallace DM Attempters, adherers, and non-adherers: latent profile analysis of CPAP use with correlates. Sleep Med. 2015;16(3):336-42. http:// dx.doi.org/10.1016/j.sleep.2014.08.013

10. Kadyan A, Asghar J, Dowson L, Sandramouli S. Ocular findings in sleep apnoea patients using continuous positive airway pressure. Eye (Lond). 2010;24(5):843-50. http://dx.doi.org/10.1038/eye.2009.212

11. Coller D, Stanley D, Parthasarathy S. Effect of air leak on the performance of auto-PAP devices: a bench study. Sleep Breath. 2005;9(4):167-75. http://dx.doi.org/10.1007/s11325-005-0032-z
In conclusion, among patients treated with CPAP, most of whom used a nasal mask, the use of a gel pillow designed to accommodate the CPAP mask did not improve treatment efficacy. However, the gel pillow was preferred over the other pillows tested, particularly by the sleepiest of our group of OSA patients. Our data do not allow us to draw conclusions in this regard. Further studies are needed in order to test this hypothesis and determine the long-term benefits of different types of pillows.

\section{ACKNOWLEDGMENTS}

The authors wish to thank Mr. Giovanni Sciortino and Mr. Fabio Artisi for their technical support.

12. Heidinger F. Report of ergonomic functional testing of the microclimatic properties (temperature) of the Pillow DELUXE COMOLD H14. Warngau: Ergonomie Institut München; 2014.

13. Young T, Finn L, Peppard PE, Szklo-Coxe M, Austin D, Nieto FJ, et al. Sleep disordered breathing and mortality: eighteen-year follow-up of the Wisconsin sleep cohort. Sleep. 2008;31(8):1071-8.

14. D'Ambrosio C, Bowman T, Mohsenin V. Quality of life in patients with obstructive sleep apnea: effect of nasal continuous positive airway pressure--a prospective study. Chest. 1999;115(1):123-9. http://dx.doi.org/10.1378/chest.115.1.123

15. Grimaldi D, Beccuti G, Touma C, Van Cauter E, Mokhlesi. Association of obstructive sleep apnea in rapid eye movement sleep with reduced glycemic control in type 2 diabetes: therapeutic implications. Diabetes Care. 2014;37(2):355-63. http://dx.doi.org/10.2337/dc130933

16. Mokhlesi B, Finn LA, Hagen EW, Young T, Hla KM, Cauter EV, et al. Obstructive sleep apnea during REM sleep and hypertension. Results of the Wisconsin Sleep Cohort. Am J Respir Crit Care Med. 2014;190(10):1158-67. http://dx.doi.org/10.1164/rccm.201406$11360 \mathrm{C}$

17. Bettinzoli M, Taranto-Montemurro L, Messineo L, Corda L, Redolfi $S$, Ferliga $M$, et al. Oronasal masks require higher levels of positive airway pressure than nasal masks to treat obstructive sleep apnea. Sleep Breath. 2014;18(4):845-9. http://dx.doi.org/10.1007/s11325 014-0954-4

18. Teo M, Amis T, Lee S, Falland K, Lambert S, Wheatley J. Equivalence of nasal and oronasal masks during initial CPAP titration for obstructive sleep apnea syndrome. Sleep. 2011;34(7):951-5. http:// dx.doi.org/10.5665/sleep. 1134

19. Bratton DJ, Stradling JR, Barbé F, Kohler M. Effect of CPAP on blood pressure in patients with minimally symptomatic obstructive sleep apnoea: a meta-analysis using individual patient data from four randomised controlled trials. Thorax. 2014;69(12):1128-35. http:// dx.doi.org/10.1136/thoraxjnl-2013-204993

20. Schwab RJ, Badr SM, Epstein LJ, Gay PC, Gozal D, Kohler M, et al. An official American Thoracic Society statement: continuous positive airway pressure adherence tracking systems. The optima monitoring strategies and outcome measures in adults. Am $J$ Respir Crit Care Med. 2013;188(5):613-20. http://dx.doi.org/10.1164/ rccm.201307-1282ST 